\title{
Combining spatial and chemical information for clustering pharmacophores
}

\author{
Lingxiao Zhou" ${ }^{*}$, Renate Griffith², Bruno Gaeta ${ }^{1}$ \\ From Asia Pacific Bioinformatics Network (APBioNet) Thirteenth International Conference on Bioinformatics \\ (InCoB2014) \\ Sydney, Australia. 31 July - 2 August 2014
}

\begin{abstract}
Background: A pharmacophore model consists of a group of chemical features arranged in three-dimensional space that can be used to represent the biological activities of the described molecules. Clustering of molecular interactions of ligands on the basis of their pharmacophore similarity provides an approach for investigating how diverse ligands can bind to a specific receptor site or different receptor sites with similar or dissimilar binding affinities. However, efficient clustering of pharmacophore models in three-dimensional space is currently a challenge.

Results: We have developed a pharmacophore-assisted Iterative Closest Point (ICP) method that is able to group pharmacophores in a manner relevant to their biochemical properties, such as binding specificity etc. The implementation of the method takes pharmacophore files as input and produces distance matrices. The method integrates both alignment-dependent and alignment-independent concepts.

Conclusions: We apply our three-dimensional pharmacophore clustering method to two sets of experimental data, including 31 globulin-binding steroids and 4 groups of selected antibody-antigen complexes. Results are translated from distance matrices to Newick format and visualised using dendrograms. For the steroid dataset, the resulting classification of ligands shows good correspondence with existing classifications. For the antigen-antibody datasets, the classification of antigens reflects both antigen type and binding antibody. Overall the method runs quickly and accurately for classifying the data based on their binding affinities or antigens.
\end{abstract}

\section{Background}

Pharmacophore methods are widely used in drug discovery research projects [1]. As defined in the International Union of Pure and Applied Chemistry (IUPAC) glossary of terms [2], a pharmacophore describes chemical features and their spatial arrangement in active molecules and targets involved in specific biochemical interactions. Several software tools provide solutions for pharmacophore modelling and generation, including Accelrys Discovery Studio [3], LigandScout [4], ZINCPharmer [5].

Pairwise comparison of pharmacophores requires defining a similarity metric. Generally, there are two categories of similarity measurements: alignment-dependent

\footnotetext{
* Correspondence: lingxiaoz@cse.unsw.edu.au

'School of Computer Science and Engineering, UNSW Australia, Sydney, NSW, Australia

Full list of author information is available at the end of the article
}

methods and alignment-independent methods [6]. Alignment-independent methods usually target binary fingerprint descriptors, such as 3-point pharmacophore fingerprints [7] or 4-point pharmacophore fingerprints [8]. They calculate similarities with measurements such as the Tanimoto similarity (also called Jaccard Index as it was originally introduced by Paul Jaccard [9]). Alignment-dependent methods [6], in most of the cases, are based on shape or shape plus pharmacophore similarity measurements. Superimposition or overlays are widely used in this category of methods. However, chemical information is typically not involved in the shape-based methods. The OpenEye [10] colour-Tanimoto is an exception. It sums overlaps using customised pharmacophore features. However, this requires painstaking manual definition of the target features. 
For grouping pharmacophores at a quantitative level, it is important to find an optimal partition method. Cluster analysis or clustering aims to separate data into groups or clusters. Clustering methods group data based on their pairwise distances. In other words, similar objects are grouped together more closely than dissimilar objects. There are some fundamental steps involved in a clustering activity including data extraction, similarity measurement, clustering and validation [11]. In cheminformatics applications, hierarchical clustering is one of the most popular approaches. These clustering methods group data based on their distances. The group average method (GA) and Ward's method [12] are two examples of hierarchical methods. Partition evaluation is a significant step to judge a clustering method. If the clustering method is applied to a benchmark dataset of known classification, then validation methods such as the Rand index and the adjusted Rand index [13] for supervised learning can be applied for comparing the results of the clustering method with the benchmark classification. Otherwise, unsupervised learning evaluation algorithms such as the Davies-Boulding index [14] can be used.

We present here a pharmacophore-aided Iterative Closest Point (ICP) clustering method for grouping pharmacophores using both their structural and chemical information. In this paper, Discovery Studio Modelling Environment accelrys.com, release 3.5 or 4.0 , is used to generate the pharmacophores. There are six features defined in Discovery studio from which to construct pharmacophore models. They are Hydrogen bond acceptor, Hydrogen bond donor, Hydrophobic, Positive ionisable (from Catalyst's definition, a "Group that is, or can be, positively charged at physiological $\mathrm{pH}$, ") [3] , Negative ionisable (from Catalyst's definition, a "Group that is, or can be, negatively charged at physiological $\mathrm{pH}$, ,) [3] and Ring aromatic (from Catalyst's definition, a "Five- or sixmembered aromatic ring (vector)") [3]. A computer vision method, Iterative Closest Point (ICP) [15], is employed to calculate pharmacophore structural distances and a greedy alignment method is applied to measure the chemical distance. These two distance measures are then combined prior to hierarchical clustering. The method is evaluated relative to existing methods using two sets of experimental data. The results demonstrate that the proposed method is not only of benefit for classification of pharmacophores, but also has the potential to facilitate research in the field of antibody-antigen interactions.

\section{Methods}

\section{Data preparation and pharmacophore generation}

Two experimental data sets were used in testing. The first set of 31 globulin binding steroids (Figure 1) was introduced by Carmer et al [15]. In recent years, this dataset has been studied using a range of clustering methods and descriptors [16-19]. We compare our proposed method to a previous study [16] that used fourpoint pharmacophores as molecular descriptors.

Antibody-antigen binding is known to be highly specific [20]. Pharmacophores, by definition [2], can describe features involved in the interaction between compounds and target. Therefore, our second evaluation involves classifying pharmacophores generated from antibody-antigen complexes. The complexes were obtained from the Protein Data Bank [21] and information about the antibodies and antigens was gathered from an online self maintaining database SACS [22]. After applying the selection criteria (human sourced antibody-antigen complexes), 207 entries were selected and aligned by Clustal Omega [23]. To simplify the evaluation, 41 complexes were selected, corresponding to 3 differently named antibodies (17B, 2F5 and Anti-HIV V3 FAB 2557) and 2 types of antigens (GP120 and GP41) (See Additional file 1). However, Discovery Studio does not accept compounds over 1000 atoms or protein as ligands. Therefore, for each of the large (over 1000 atoms) protein antigens, the compound had to be cut into several parts and be saved in molecule format (SD file format). The cutting was based on the potential contact surface on the antigens. The potential contact surfaces were determined by finding the neighbouring (distance equal or less than $2.5 \AA$ ) amino acids of the antibody chains.

Discovery Studio Modelling Environment, release 4.0, generates the pharmacophores as *.chm files. Several protocols were employed for generating the pharmacophores. The autopharmacophore generation protocol selects pharmacophores using a Genetic Function Approximation (GFA) model [24]. This protocol aims to generate pharmacophore models from a single input molecule. Thirty one pharmacophores were generated using this protocol. The pharmacophore details for the globulin binding steroids have been recorded (See Additional file 2). For proteinligand interactions, the GFA model as coded in the receptor-ligand pharmacophore generation protocol was used to produce structure-based pharmacophore models. Antibody-small molecules and antibody-protein parts were processed using this protocol. The details of the 41 antibody-antigen complex pharmacophores are listed in the table in Additional file 3. In this table, partial pharmacophores for large protein antigens were combined. The combination details are explained in the next section.

\section{Parsing pharmacophore files}

The pharmacophore files produced by Discovery Studio include information such as name, coordinates, vector and tolerance etc. of the pharmacophore features. Based on our method, a set of Perl scripts were written to perform a series of steps to phase the pharmacophore files. Structural and chemical information was extracted from pharmacophore files. To simplify the calculation, some vector 


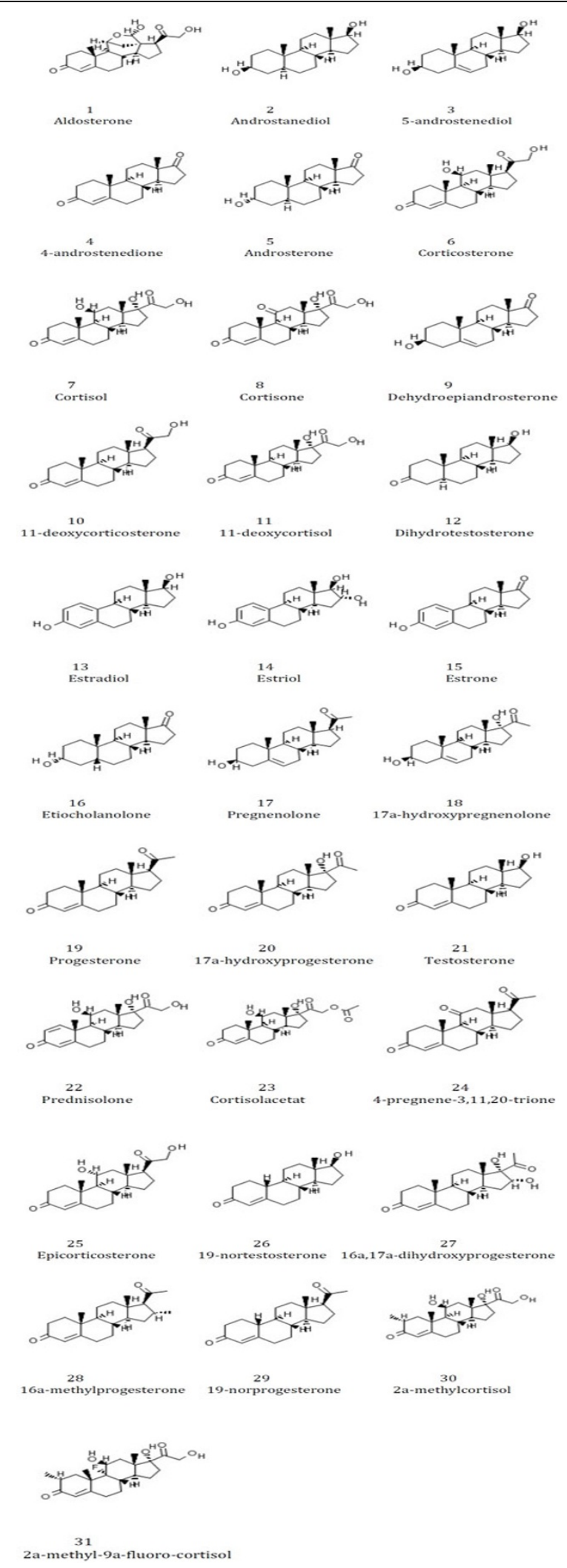

Figure $12 \mathrm{D}$ molecular structures and names of the 31 globulin binding steroids. 
features, such as hydrogen bond donors and hydrogen bond acceptors were represented as one point. The coordinates of this point were provided by the centroid of the vector. Some statistics for each pharmacophore model were calculated and recorded, including the name of the features for each model, feature counters for each feature and so on. In the final stage of the phasing, the centroids of all pharmacophore models were normalized to $(0,0,0)$, and new coordinates were calculated.

\section{ICP based structural distance calculation}

The clustering was implemented in Matlab using the Iterative Closest Point (ICP) algorithm. ICP $[15,25]$ is a method for optimizing the sum of squared distances between two sets of points. It is widely used in the fields of computer vision and robot navigation. The following is a summary of the ICP algorithm we implemented. It calculates the 3D structural information of two pharmacophores $p$ and $q$ to generate a rotation matrix $\mathrm{R}$ and a translation matrix $\mathrm{T}$.

For $\mathrm{k}=1$ to $\mathrm{k}_{\max }$

1. Do selection and matching Build k-d tree[26] and find closest neighbor pairs with KNN search 2. If matches to edge vertices or worst matches detected Do rejection point pairs

3. Weight matched points Weighting with compatibility of normal:

$\mathrm{W}=\mathrm{n}_{p} * \mathrm{n}_{q}$
4. Minimize the error metric Calculate $\mathrm{R}$ with singular value decomposition (SVD)[27]:

$$
\mathrm{R}=\mathrm{V} * \mathrm{U}^{\mathrm{T}}
$$

Calculate T:

$$
\mathrm{T}=\bar{q}-R * \bar{p}
$$

\section{End for}

5. Assign and apply transformation

Figure 2. demonstrates this implementation by applying the ICP algorithm to our antibody-antigen dataset. Blue points represent the template set, the green and red points represent the second set, with the green points representing the initial pharmacophore locations and the red points representing them after application of the transformation.

The structural distance of the two pharmacophores was calculated using the Root-mean-square deviation (RMSD). RMSD values were normalized by dividing by the maximum distance. In the end, a $\mathrm{N}^{*} \mathrm{~N}$ structural distance matrix was produced based on the number of pharmacophore models $(\mathrm{N})$.

\section{Greedy alignment-based chemical distance calculation}

The second significant part of the method is to compute a chemical distance matrix. A greedy alignment method was introduced to compare the chemical differences between pharmacophore models. This alignment approach was coded in Matlab like the ICP algorithm.

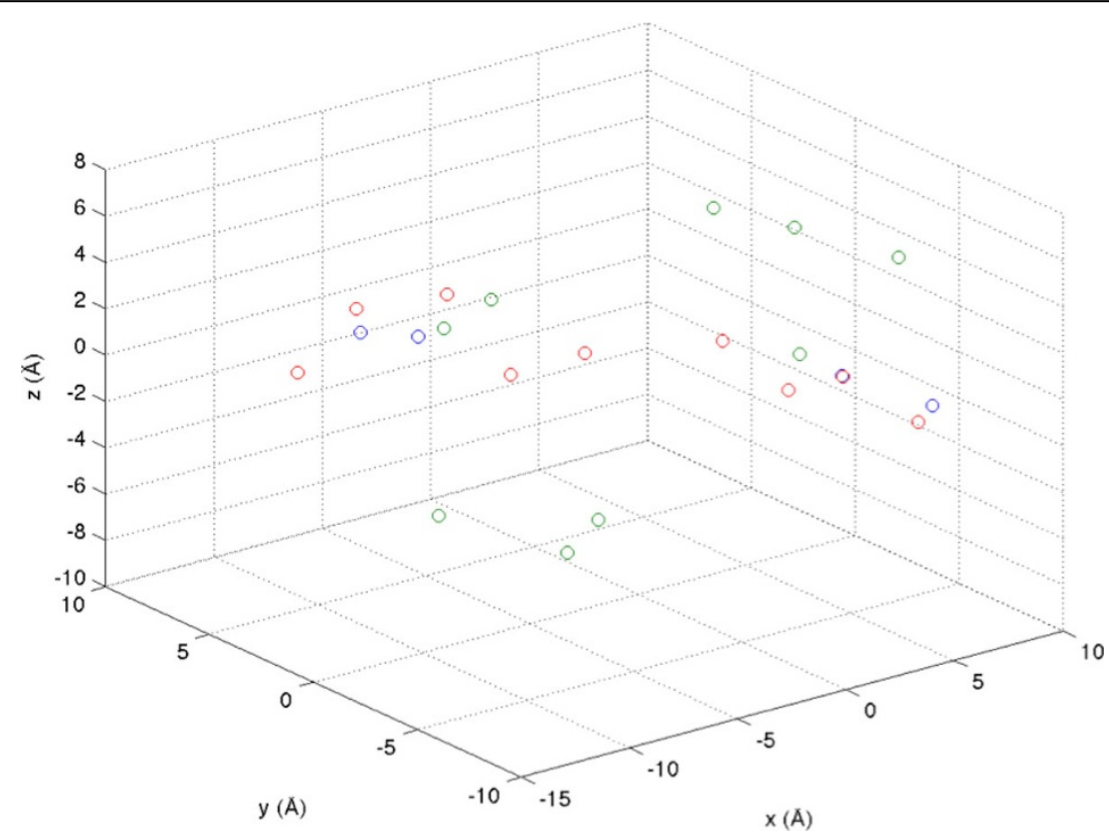

Figure 2 ICP application to two antigens from PDB entries 1ADQ_P2 [33] and 3GBN [34]. 1ADQ_P2 is shown in blue and is the reference model. Green points represent 3GBN before application of ICP. Red points correspond to 3GBN after ICP transformation based on 1ADQ_P2. 
In this method, a pharmacophore scoring matrix, as used in the Pharmacophore Alignment Search Tool (PhAST) [28], played an important role. The procedure of the greedy alignment is as follows. Let us consider two pharmacophore lists $\left\{p_{\mathrm{i}}\right\}$ (pharmacophores 1) and $\left\{q_{j}\right\}$ (pharmacophores 2). $n$ is the number of features in $\left\{p_{\mathrm{i}}\right\}$ and $m$ is the number of features in $\left\{q_{\mathrm{j}}\right\}$.

1. Find common features from both groups and remove them

2. Find the "best-unmatched" (feature pair with lowest dissimilarity score) features

a. Remove them

b. Increase the penalty score

3. Calculate gaps $(|n-m|)$

a. Increase the penalty score

The chemical distance matrix was calculated for each possible pair of pharmacophores. The matrix was then normalized by the maximum value of the gap penalty (by dividing each value in the matrix by the gap penalty $\left.{ }^{*} \max (n, m)\right)$. A gap penalty score of 14 per position was used in the calculation, as in the PhAST method [28].

\section{Combined distance matrix}

In the final step of the method, the structural distance matrix and the chemical distance matrix were integrated to form a mixed distance matrix. The combined matrix includes a geometric term $\mathrm{S}$ and a chemical term $\mathrm{C}$ :

$$
\mathrm{D}=\lambda * \mathrm{~S}+(1-\lambda) * \mathrm{C}
$$

In equation (4), $\lambda$ can be adjusted to change the weights of $3 \mathrm{D}$ and chemical data. The workflow for the complete procedure can be found in Figure 3.

\section{Results}

\section{Globulin-binding steroids}

After applying our clustering method, a $31 * 31$ distance matrix was generated. The tree (Figure 4) was created using T-REX [29] from the combined matrix and using the neighbour joining method. This tree was compared with trees produced from the same dataset by two other methods [16]. One of the trees (Figure 5) was generated

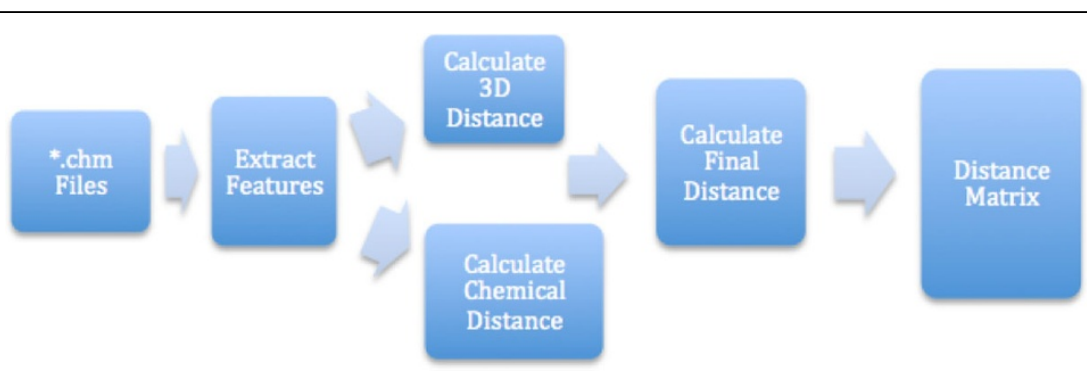

Figure 3 Workflow of the ICP aided pharmacophore clustering method

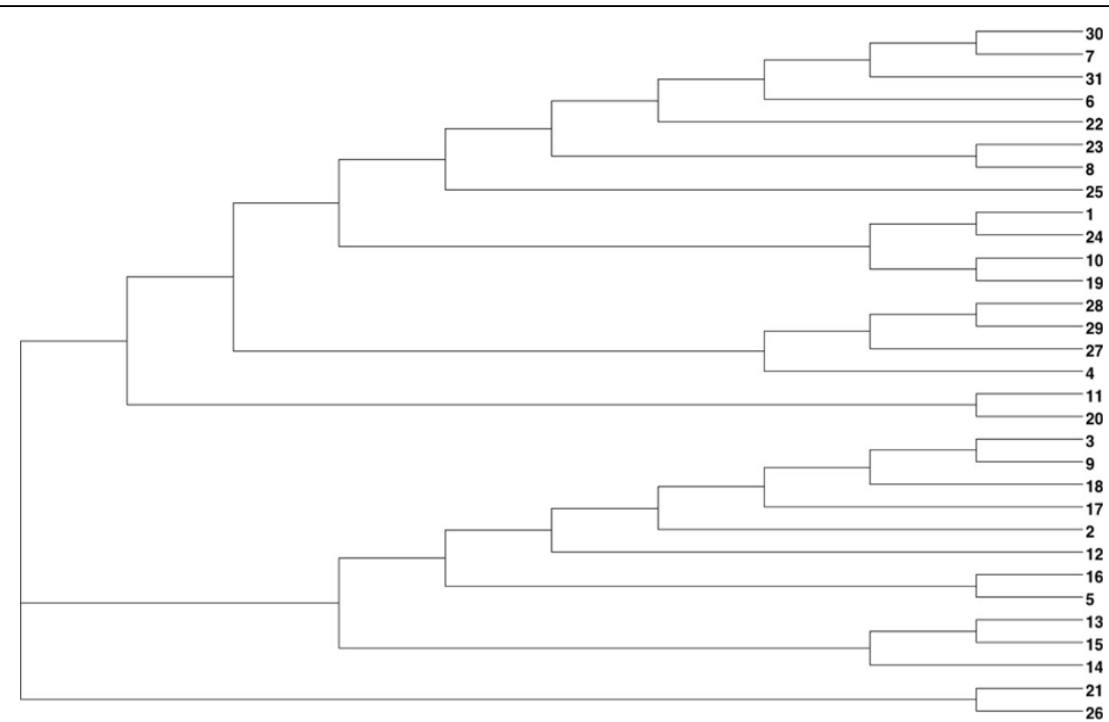

Figure 4 Clustering of the $\mathbf{3 1}$ globulin binding steroids. This dendrogram is showing the clustering of the 31 globulin binding steroids derived using a combination of $3 \mathrm{D}$ and chemical distances. 


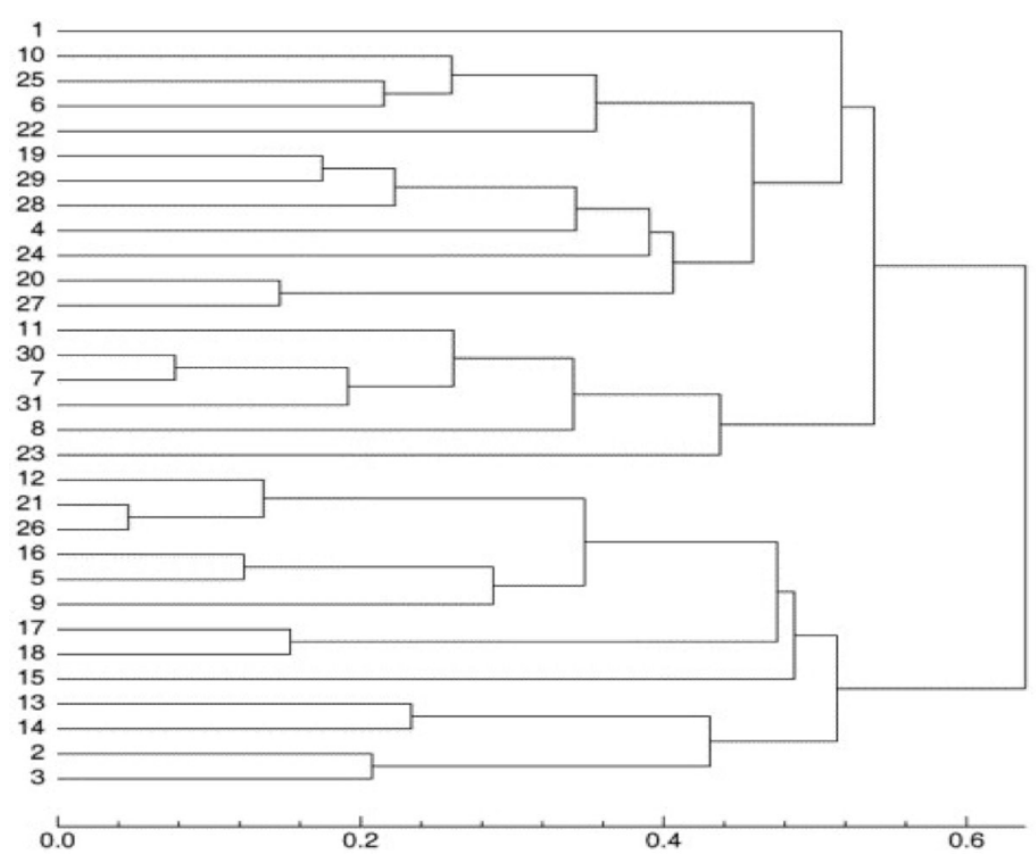

Figure 5 Clustering of the $\mathbf{3 1}$ globulin binding steroids. This dendrogram is showing the clustering of the 31 globulin binding steroids derived using the group average clustering method by Rodriguez [16].

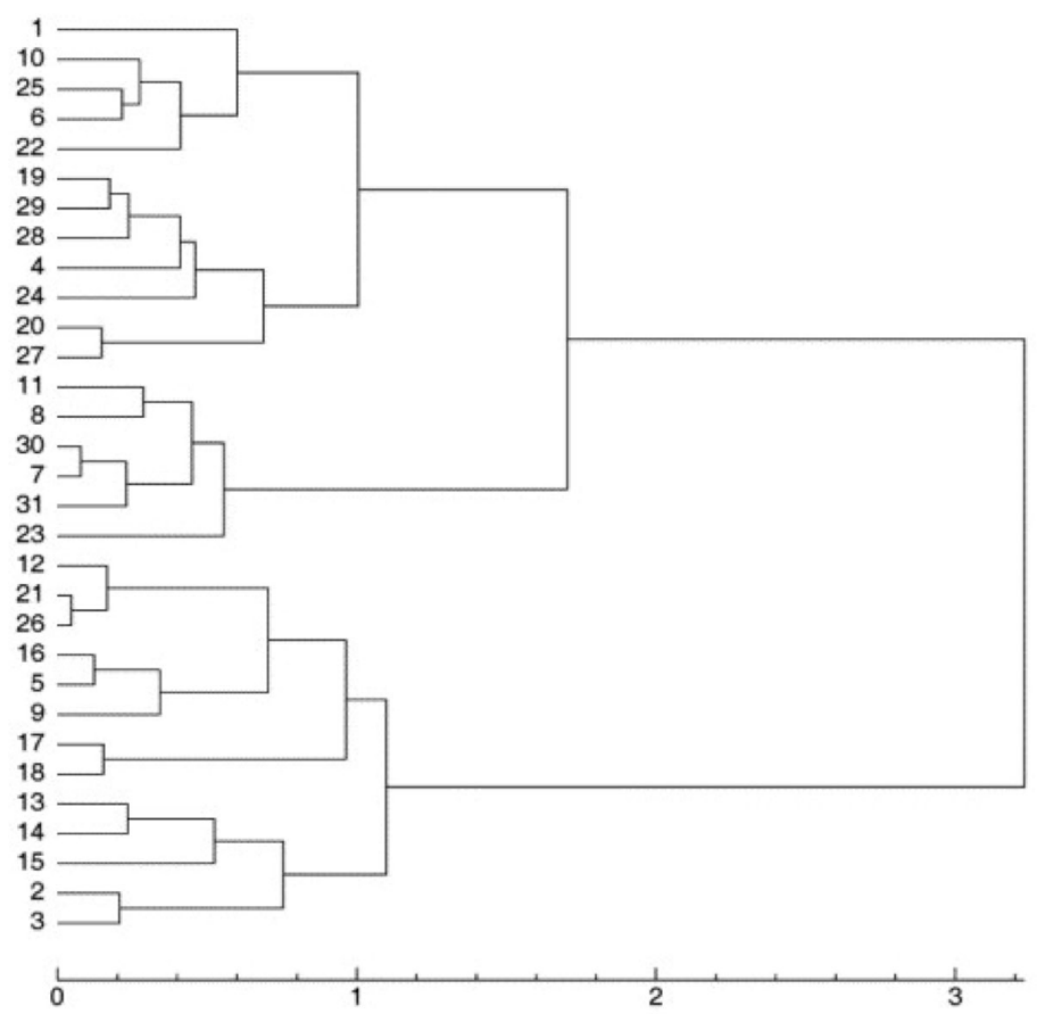

Figure 6 Dendrogram showing the clustering of the 31 globulin binding steroids derived using Ward's clustering method by Rodriguez [16]. This dendrogram is showing the clustering of the 31 globulin binding steroids derived using Ward's clustering method by Rodriguez [16]. 
with the group average method [30], and the other one (Figure 6) was derived using Ward's method [12].

For further comparison, a table of binding affinity information for the 31 molecules from the literature [31] is provided as a gold standard to evaluate all three methods (Table 1). The 31 molecules were divided into two groups based on this binding affinity data: group 1 $(\mathrm{CGB}<-6.2)$ and group 2 (CGB>-6.2), to provide a reference clustering (Table 2). For the clusterings produced by Rodriguez and that produced by our method, the 31 compounds were also labeled based on the clustering results (Table 2). Both clusterings were then compared to the reference CBG clustering using the Rand Index and adjusted Rand Index methods [13]. The evaluation results are shown in Table 3. All methods performed equally well in recreating the benchmark clustering.

\section{Antibody-antigen complex dataset}

In this section, the ICP-based pharmacophore-aided method was applied to classify 4 groups of pharmacophores. The clustering method generated a $41^{*} 41$ distance matrix. T-REX translated the distance matrix into a dendrogram (Figure 7).

To evaluate the result, we categorised the 41 complexes into two groups based on their antigens, as a benchmark clustering. Results from the new method were clustered into 4 groups (Figure 7 and Additional file 1). There were two large clusters G1 (antigen GP41),

Table 1 Binding affinities of the 31 globulin binding steroids [31].

\begin{tabular}{|c|c|c|}
\hline Molecule & ID & CBG $\left(\mathrm{pK}_{\mathrm{a}}\right)$ \\
\hline Deoxycortisol & 11 & -7.881 \\
\hline Corticosterone & 6 & -7.881 \\
\hline Cortisol & 7 & -7.881 \\
\hline Hydroxyprogesterone & 20 & -7.740 \\
\hline 2a-methylcortisol & 30 & -7.688 \\
\hline Deoxycorticosterone & 10 & -7.653 \\
\hline Cortisolacetate & 23 & -7.553 \\
\hline Prednisolone & 22 & -7.512 \\
\hline Progesterone & 19 & -7.380 \\
\hline Epicorticosterone & 25 & -7.200 \\
\hline 17a-methylprogesterone & 28 & -7.120 \\
\hline Cortisone & 8 & -6.892 \\
\hline 19-Norprogesterone & 29 & -6.817 \\
\hline 4-Pregnene-3,11,20-trione & 24 & -6.779 \\
\hline Testosterone & 21 & -6.724 \\
\hline Aldosterone & 1 & -6.279 \\
\hline 16a,17a-Dihydroxyprogesterone & 27 & -6.247 \\
\hline 19-Nortestosterone & 26 & -6.144 \\
\hline Dihydrotestosterone & 12 & -5.919 \\
\hline 2a-methyl-9a-fluorocortisol & 31 & -5.797 \\
\hline 4-Androstenedion & 4 & -5.763 \\
\hline Androsterone & 5 & -5.613 \\
\hline Pregnenolone & 17 & -5.225 \\
\hline Etiocholanolone & 16 & -5.225 \\
\hline Androstanediol & 2 & -5.000 \\
\hline 5-Androstenediol & 3 & -5.000 \\
\hline Dehydroepiandrosterone & 9 & -5.000 \\
\hline Estradiol & 13 & -5.000 \\
\hline Estriol & 14 & -5.000 \\
\hline Estrone & 15 & -5.000 \\
\hline Hydroxypregenolone & 18 & -5.000 \\
\hline
\end{tabular}


Table 2 Group labeling for 31 globulin binding steroids.

\begin{tabular}{|c|c|c|c|c|}
\hline ID & CGB Grouping & R_GA & R_Ward & 3D Clustering \\
\hline 1 & 1 & 1 & 1 & 1 \\
\hline 2 & 2 & 2 & 2 & 2 \\
\hline 3 & 2 & 2 & 2 & 2 \\
\hline 4 & 2 & 1 & 1 & 1 \\
\hline 5 & 2 & 2 & 2 & 2 \\
\hline 6 & 1 & 1 & 1 & 1 \\
\hline 7 & 1 & 1 & 1 & 1 \\
\hline 8 & 1 & 1 & 1 & 1 \\
\hline 9 & 2 & 2 & 2 & 2 \\
\hline 10 & 1 & 1 & 1 & 1 \\
\hline 11 & 1 & 1 & 1 & 1 \\
\hline 12 & 2 & 2 & 2 & 2 \\
\hline 13 & 2 & 2 & 2 & 2 \\
\hline 14 & 2 & 2 & 2 & 2 \\
\hline 15 & 2 & 2 & 2 & 2 \\
\hline 16 & 2 & 2 & 2 & 2 \\
\hline 17 & 2 & 2 & 2 & 2 \\
\hline 18 & 2 & 2 & 2 & 2 \\
\hline 19 & 1 & 1 & 1 & 1 \\
\hline 20 & 1 & 1 & 1 & 1 \\
\hline 21 & 1 & 2 & 2 & 3 \\
\hline 22 & 1 & 1 & 1 & 1 \\
\hline 23 & 1 & 1 & 1 & 1 \\
\hline 24 & 1 & 1 & 1 & 1 \\
\hline 25 & 1 & 1 & 1 & 1 \\
\hline 26 & 2 & 2 & 2 & 3 \\
\hline 27 & 1 & 1 & 1 & 1 \\
\hline 28 & 1 & 1 & 1 & 1 \\
\hline 29 & 1 & 1 & 1 & 1 \\
\hline 30 & 1 & 1 & 1 & 1 \\
\hline 31 & 2 & 1 & 1 & 1 \\
\hline
\end{tabular}

The labels for the 31 globulin binding steroids are based on CBG (binding affinity), Group Average (R_GA), Ward (R_Ward) (Rodriguez [16]) and the combined 3D distance and chemical distance method proposed in this study (3D Clustering).

G2 (antigen GP120). Complexes 3D0L and 3D0V were misclassified, so we labelled them as G3 (3D0L) and G4 (3D0V). These two classifications were compared using the Rand Index and Adjusted Rand Index. The results (Table 4) demonstrate an excellent agreement between the two classifications.

\section{Discussion}

In the dataset of 31 steroid compounds, some pairs had been reported that should be grouped together closely ([16]). They were $(21,26),(7,30)$ and $(19,29)$, that differ only by a methyl group. Molecules 5 and 16 only differ by the stereochemistry of one centre on the A ring. Comparison of Figures 4, 5 and 6 demonstrate that all three

Table 3 Evaluation of different clustering methods for 31 globulin binding steroids.

\begin{tabular}{clll}
\hline Method & Rand Index (0 to 1) & Adjusted RI (-1 to 1) \\
\hline R_GA & 0.8194 & 0.6387 \\
\hline R_Ward & 0.8194 & 0.6387 \\
\hline 3D Clustering & 0.8194 & 0.6378 \\
\hline
\end{tabular}

Rand index and adjusted Rand index are given for the comparison of each of the clustering methods with the reference CBG clustering for 31 globulin binding steroids. 


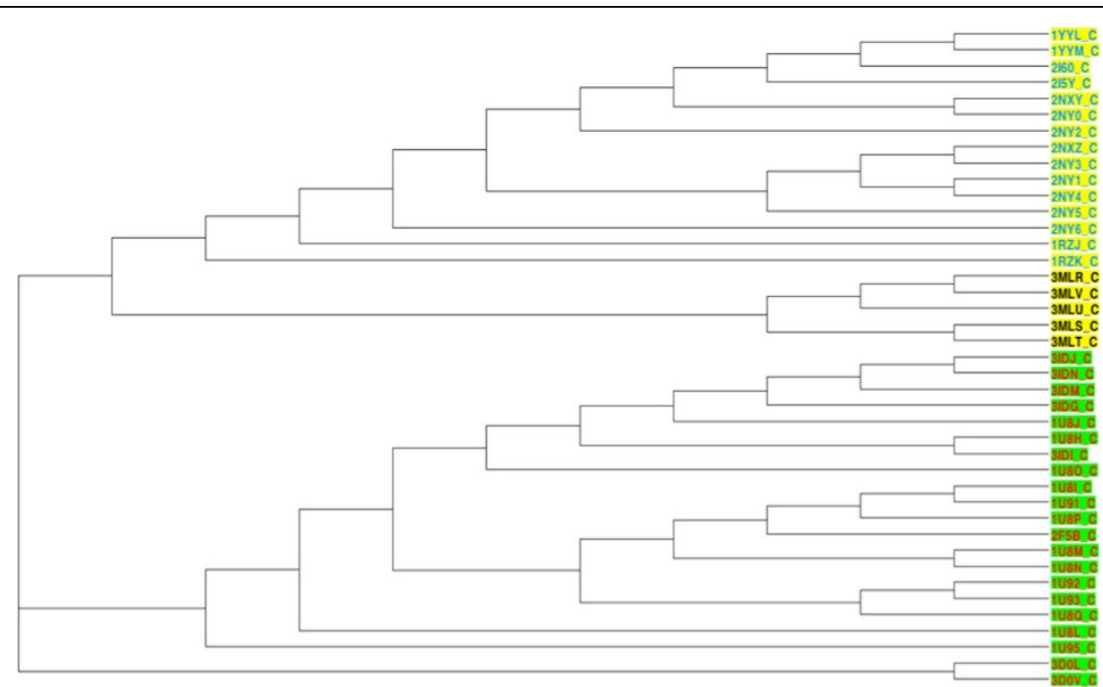

Figure $\mathbf{7}$ Clustering of $\mathbf{4 1}$ antibody-antigen complexes based on combined distance. This dendrogram is showing the clustering of four groups of antibody-antigen complexes based on a combination of 3D and chemical distances. Complexes with antigen GP41 are shown with a green background. Complexes with antigen GP120 are shown with a yellow background. Complexes with antibody 17B, are shown with their PDB ID colored blue. Complexes with antibody 2F5 are shown with their PDB ID colored red. Complexes with antibody ANTI-HIV-1 V3 FAB 2557 are shown with their PDB ID colored black.

Table 4 Evaluation of 3D plus chemical clustering method for antibody-antigen complexes.

\begin{tabular}{ccc}
\hline Method & Rand Index (0 to 1) & Adjusted RI (-1 to 1) \\
\hline 3D Clustering & 0.9524 & 0.9046 \\
\hline
\end{tabular}

Rand index and adjusted Rand index results for comparison of the 3D plus chemical clustering method with the reference clustering based on antigen identity for 41 antibody-antigen complexes.

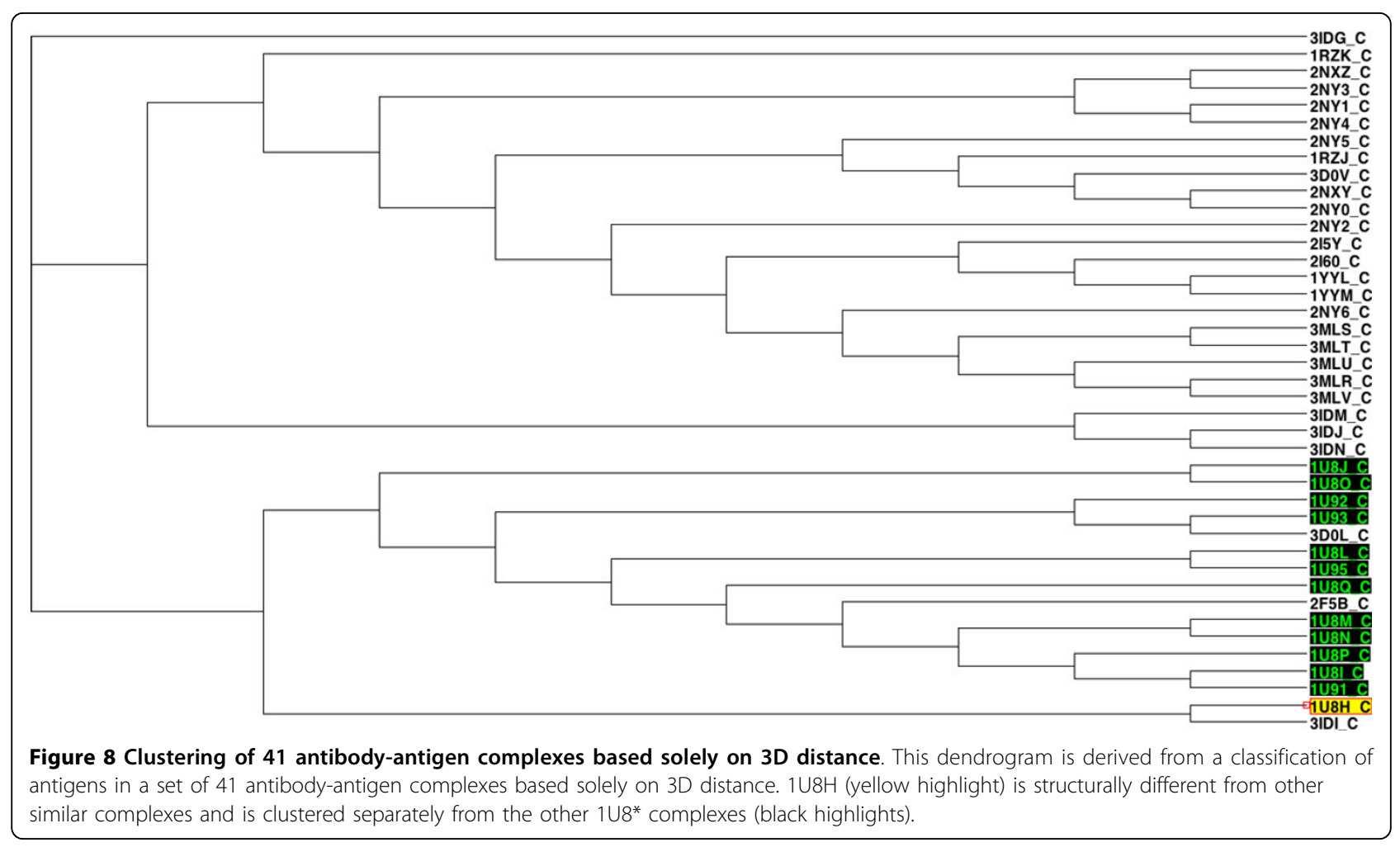




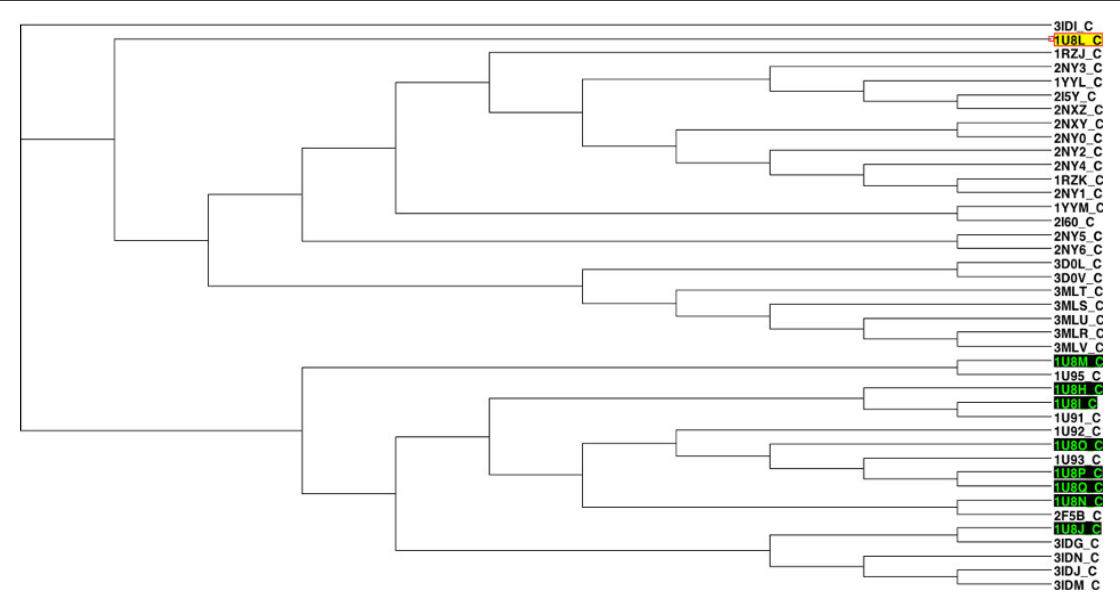

Figure 9 Clustering of $\mathbf{4 1}$ antibody-antigen complexes based solely on chemical distance. This is a dendrogram based on a classification of antigens in a set of 41 antibody-antigen complexes based solely on chemical differences between pharmacophores. 1U8L (yellow highlight) chemically differs from other similar complexes and is clustered away from the other 1 U $8^{*}$ complexes (black highlights).

different methods have successfully grouped those reported pairs. The special structures of the two compounds 4 and 31 led to a misclassification (they were classified into group with $\mathrm{pKa}<-6.2)$ in all three methods. Molecules 21 and 26 were incorrectly clustered as an exceptional cluster by our new method. With the exception of those molecules (21 and 26), the group average method, Ward's method and our method all produced trees with the same two superclasses. Rodriguez's methods and the new method have the same Rand Index value and a very close adjusted Rand Index. Additionally, all Rand Index and adjusted Rand Index scores are above the threshold for a 'good' clustering ( 0.5 for Rand Index, 0 for adjusted Rand Index).

Considering the application of the proposed method to 41 antibody-antigen complexes, the pharmacophores were generally classified into two large super-clusters based on their antigens. One supercluster included all complexes with GP41 or a GP41 analog as antigen. The second supercluster had all the complexes with GP120 or one of its fragments as antigen. The classification did not only identify the antigens, within each supercluster, pharmacophores also formed clusters corresponding to their binding antibody (e.g. G1 with 17B as antibody and G4 with ANTI-HIV-1 V3 FAB 2557). Additionally, the Rand Index and adjusted Rand Index both were very high, which means the ICP aided method performed well in clustering. In addition some interesting structural and chemical features highlighted by other researchers could be identified in the results. In complex $1 \mathrm{U} 8 \mathrm{H}$, the Glu662 substitution has been reported to involve a water network rearrangement and thus this complex is structurally different from the other 1U8* complexes [32]. This can be seen by the unexpected position of $1 \mathrm{U} 8 \mathrm{H}$ in a clustering based solely

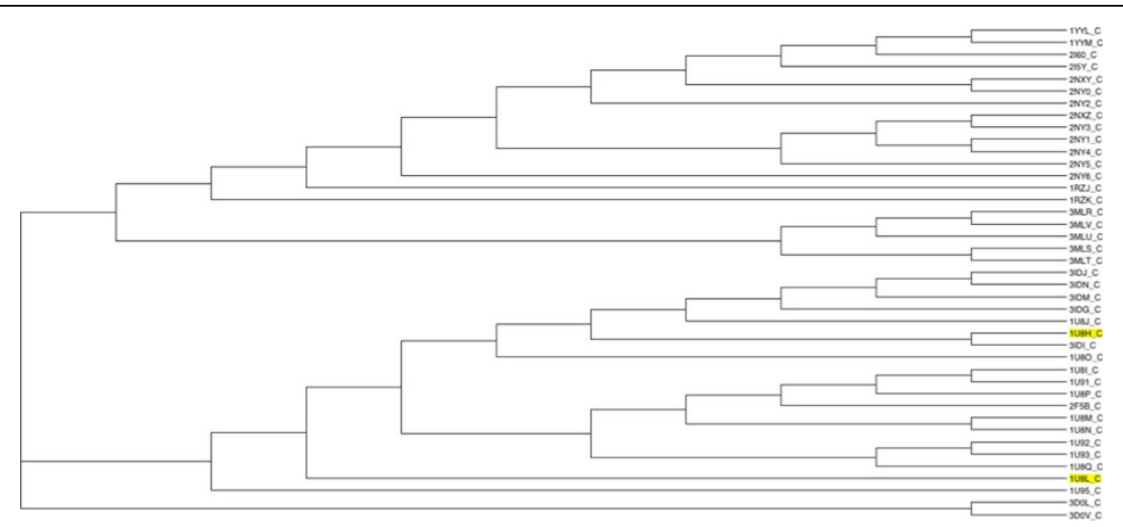

Figure 10 Clustering of $\mathbf{4 1}$ antibody-antigen complexes based on combined distance. This section of a dendrogram calculated from a distance measure combining 3D structural distance and chemical distance between pharmacophores. 1U8L and 1U8H (highlighted in yellow) are correctly identified based on antigen. 
on 3D distance calculated using ICP (Figure 8). In the same paper, $1 \mathrm{U} 8 \mathrm{~L}$ was reported to have chemical differences to the other $1 \mathrm{U}^{*}$ complexes. This can be seen on a dendrogram based solely on chemical distances (Figure 9). However, when 3D and chemical distances were combined, 1U8L and 1U8H were correctly clustered with other complexes with similar antigens (Figure 10).

\section{Conclusions}

A method combining a structural distance based on ICP and a "chemical" distance has been developed and has been demonstrated to successfully partition pharmacophores based on the types of antigens in a set of antibody/ antigen complexes or binding affinity in a set of steroids. In addition, the method is very fast. The 41 pharmacophore comparison only took around 30 seconds on a desktop computer (Apple iMac, $2.7 \mathrm{GHz}$ Intel Core i5, 8GB Memory). However, the method requires the number of pharmacophores being compared to be similar and was less accurate when the following ratio was larger than 2.: Max(Number_of_Pharmacophores)/Min (Number_of_Pharmacophores)

\section{Additional material}

Additional file 1: Antibody-antigen complexes. This table summarises antibody-antigen complexes used in this study with their cluster number as assigned by the ICP-based method.

Additional file 2: Number of pharmacophore features in the 31 globulin binding steroids used in this study

Additional file 3: Number of pharmacophore features in the 41 antibody-antigen complexes used in this study

\section{Competing interests}

The authors declare that they have no competing interests.

Authors' contributions

All authors read and approved the final manuscript.

\section{Acknowledgements}

The publication costs for this article were funded from a grant from the School of Computer Science and Engineering, UNSW Australia. This article has been published as part of BMC Bioinformatics Volume 15 Supplement 16, 2014: Thirteenth International Conference on Bioinformatics (InCoB2014): Bioinformatics. The full contents of the supplement are available online at http://www.biomedcentral.com/bmcbioinformatics/ supplements/15/S16

\section{Authors' details}

${ }^{1}$ School of Computer Science and Engineering, UNSW Australia, Sydney, NSW, Australia. ${ }^{2}$ School of Medical Sciences/Pharmacology, UNSW Australia, Sydney, NSW, Australia.

Published: 8 December 2014

\section{References}

1. Leach AR, Gillet VJ, Lewis RA, Taylor R: Three-Dimensional Pharmacophore Methods in Drug Discovery. J Med Chem 2010, 53(2):539-558.

2. Wermuth $G$, Ganellin CR, Lindberg P, Mitscher LA: Glossary of terms used in medicinal chemistry (IUPAC Recommendations 1998). Pure Appl Chem 1998, 70(5):1129-1143.
3. Sutter J, Li JB, Maynard AJ, Goupil A, Luu T, Nadassy K: New Features that Improve the Pharmacophore Tools from Accelrys. Curr Comput-Aid Drug 2011, 7(3):173-180.

4. Wolber G, Dornhofer AA, Langer T: Efficient overlay of small organic molecules using 3D pharmacophores. Journal of computer-aided molecular design 2006, 20(12):773-788.

5. Koes DR, Camacho CJ: ZINCPharmer: pharmacophore search of the ZINC database. Nucleic Acids Res 2012, 40(W1):W409-W414.

6. MacCuish JD, MacCuish NE: Chemoinformatics applications of cluster analysis. Wires Comput Mol Sci 2014, 4(1):34-48.

7. Good AC, Kuntz ID: Investigating the Extension of Pairwise Distance Pharmacophore Measures to Triplet-Based Descriptors. Journal of computer-aided molecular design 1995, 9(4):373-379.

8. Mason JS, Cheney DL: Library design and virtual screening using multiple 4-point pharmacophore fingerprints. Pac Symp Biocomput 2000, 576-587.

9. Jaccard P: The distribution of the flora in the alpine zone. New Phytologist 1912, 11(2):37-50.

10. Hawkins PCD, Skillman AG, Nicholls A: Comparison of shape-matching and docking as virtual screening tools. J Med Chem 2007, 50(1):74-82.

11. Jain AK, Murty MN, Flynn PJ: Data clustering: A review. Acm Comput Surv 1999, 31(3):264-323.

12. Ward JH: Hierarchical Grouping to Optimize an Objective Function. J Am Stat Assoc 1963, 58(301):236-\&.

13. Hubert L, Arabie P: Comparing Partitions. J Classif 1985, 2(2-3):193-218.

14. Davies DL, Bouldin DW: Cluster Separation Measure. leee T Pattern Ana 1979, 1(2):224-227

15. Besl PJ, Mckay ND: A Method for Registration of 3-D Shapes. leee T Pattern Anal 1992, 14(2):239-256.

16. Rodriguez A, Tomas MS, Perez JJ, Rubio-Martinez J: Assessment of the performance of cluster analysis grouping using pharmacophores as molecular descriptors. J Mol Struc-Theochem 2005, 727(1-3):81-87.

17. Cramer RD, Patterson DE, Bunce JD: Comparative Molecular-Field Analysis (Comfa) .1. Effect of Shape on Binding of Steroids to Carrier Proteins. J Am Chem Soc 1988, 110(18):5959-5967.

18. Wagener M, Sadowski J, Gasteiger J: Autocorrelation of Molecular-Surface Properties for Modeling Corticosteroid-Binding Globulin and Cytosolic Ah Receptor Activity by Neural Networks. J Am Chem Soc 1995, 117(29):7769-7775.

19. Bultinck P, Carbo-Dorca R: Molecular quantum similarity matrix based clustering of molecules using dendrograms. J Chem Inf Comp Sci 2003, 43(1):170-177.

20. Ramos-Vara JA: Technical aspects of immunohistochemistry. Vet Pathol 2005, 42(4):405-426.

21. Berman HM, Westbrook J, Feng Z, Gilliland G, Bhat TN, Weissig H, Shindyalov IN, Bourne PE: The Protein Data Bank. Nucleic Acids Res 2000 28(1):235-242

22. Allcorn LC, Martin ACR: SACS - Self-maintaining database of antibody crystal structure information. Bioinformatics 2002, 18(1):175-181.

23. Sievers F, Wilm A, Dineen D, Gibson TJ, Karplus K, Li WZ, Lopez R, McWilliam H, Remmert M, Soding J, et al: Fast, scalable generation of high-quality protein multiple sequence alignments using Clustal Omega. Mol Syst Biol 2011, 7.

24. Rogers D, Hopfinger AJ: Application of Genetic Function Approximation to Quantitative Structure-Activity-Relationships and Quantitative Structure-Property Relationships. J Chem Inf Comp Sci 1994, 34(4):854-866.

25. Chen Y, Medioni G: Object Modeling by Registration of Multiple Range Images. 1991 leee International Conference on Robotics and Automation 1991, 1-3:2724-2729.

26. Dandamudi SP, Sorenson PG: An Empirical Performance Comparison of Some Variations of the K-D Tree and Bd Tree. Int J Comput Inf Sci 1985, 14(3):135-159.

27. Alter $\mathrm{O}$, Brown PO, Botstein D: Singular value decomposition for genomewide expression data processing and modeling. Proc Natl Acad Sci USA 2000, 97(18):10101-10106.

28. Hahnke V, Hofmann B, Grgat T, Proschak E, Steinhilber D, Schneider G: PhAST: pharmacophore alignment search tool. Journal of computational chemistry 2009, 30(5):761-771.

29. Alix B, Boubacar DA, Vladimir M: T-REX: a web server for inferring, validating and visualizing phylogenetic trees and networks. Nucleic Acids Res 2012, 40(W1):W573-W579. 
30. Lohse-Bossenz H, Kunina-Habenicht O, Kunter M: Estimating within-group agreement in small groups: A proposed adjustment for the average deviation index. Eur J Work Organ Psy 2014, 23(3):456-468.

31. Robert $\mathrm{D}$, Amat L, Carbo-Dorca R: Three-dimensional quantitative structure-activity relationships from tuned molecular quantum similarity measures: Prediction of the corticosteroid-binding globulin binding affinity for a steroid family. J Chem Inf Comp Sci 1999, 39(2):333-344.

32. Bryson S, Julien JP, Hynes RC, Pai EF: Crystallographic definition of the epitope promiscuity of the broadly neutralizing antihumanimmunodeficiency virus type 1 antibody $2 F 5$ : vaccine design implications. J Virol 2009, 83(22):11862-11875.

33. Corper AL, Sohi MK, Bonagura VR, Steinitz M, Jefferis R, Feinstein A, Beale D, Taussig MJ, Sutton BJ: Structure of human IgM rheumatoid factor Fab bound to its autoantigen lgG Fc reveals a novel topology of antibodyantigen interaction. Nat Struct Biol 1997, 4(5):374-381.

34. Ekiert DC, Bhabha G, Elsliger MA, Friesen RHE, Jongeneelen M, Throsby M, Goudsmit J, Wilson IA: Antibody Recognition of a Highly Conserved Influenza Virus Epitope. Science 2009, 324(5924):246-251.

doi:10.1186/1471-2105-15-S16-S5

Cite this article as: Zhou et al:: Combining spatial and chemical information for clustering pharmacophores. BMC Bioinformatics 2014 15(Suppl 16):S5.

\section{Submit your next manuscript to BioMed Central and take full advantage of:}

- Convenient online submission

- Thorough peer review

- No space constraints or color figure charges

- Immediate publication on acceptance

- Inclusion in PubMed, CAS, Scopus and Google Scholar

- Research which is freely available for redistribution

Submit your manuscript at www.biomedcentral.com/submit 